\title{
GES en cáncer de ovario epitelial: un avance sanitario necesario pero no exento de riesgos y dificultades futuras
}

A nivel mundial el cáncer de ovario epitelial constituye la neoplasia maligna ginecológica más letal. Anualmente se diagnostican poco menos de 225.000 nuevos casos y poco más de 140.000 mujeres mueren por esta causa en el mismo período $(1,2)$. Su gran letalidad se debe mayoritariamente a que no existen herramientas de tamizaje ni síntomas premonitores que permitan su diagnóstico en etapa precoz. Así, lamentablemente, dos tercios de los casos se diagnostican en etapa avanzada (etapa III o IV de la clasificación FIGO), estado en el cual la sobrevida no supera el $40 \%$ a 5 años (3). Contribuye además a esta alta letalidad, la transitoriedad de la respuesta terapéutica que se observa en la mayoría de los casos, fenómeno que se sustenta en el alto porcentaje de recurrencias que tienen lugar a corto andar de finalizado el tratamiento (habitualmente entre 12 y 24 meses post terapia). De hecho, aunque el 70 a $80 \%$ de los casos responde adecuadamente, experimentando remisión clínica, la mayoría así también recurre en una forma de cáncer más agresiva y resistente que indefectiblemente conduce a la muerte $(3,4)$.

En la actualidad las alternativas de tratamiento para este cáncer contemplan, como primera opción, el uso de cirugía de citoreducción óptima seguida de quimioterapia. Alternativamente, para aquellos casos en que la condición clínica de la paciente no lo permite, está disponible la opción de iniciar tratamiento con quimioterapia neoadyuvante seguido de cirugía de intervalo (3-6). En ambos escenarios, se trata de un tratamiento de alta complejidad que demanda recursos humanos, económicos y de infraestructura que para países emergentes o en vías de desarrollo no siempre es posible ofrecer o en la práctica conlleva acceso restringido. Desde un punto de vista social, es una condición predispuesta a la inequidad, ya que la limitación de recursos humanos y económicos, propia de los países en vías de desarrollo, puede hacer que los beneficiarios del sistema público no accedan a toda la gama de opciones terapéuticas cuando se compara con aquellos que, contando con los medios, logran acceder al sistema de atención privada o de libre elección.

En Chile, tanto la incidencia como la mortalidad por cáncer de ovario epitelial han ido paulatinamente aumentando en la última década. A modo de ejemplo como muestra la Figura 1, la tasa de mortalidad ha aumentado desde 3,4 a 4,9 por 100.000 mujeres/año si se compara los años 1997 y 2010. Así, el cáncer de ovario epitelial ha pasado a ocupar desde el décimo al noveno lugar, como causa de muerte por cáncer en la mujer chilena, en los últimos 5 años. Tal tendencia de los indicadores se explica por el aumento en la esperanza de vida en la mujer chilena (al tratarse de una condición cuya prevalencia aumenta después de la sexta década de la vida), a la mayor prevalencia de factores de riesgo predisponentes al desarrollo de esta condición (ej. obesidad y tabaquismo) y a la variabilidad en los estándares de tratamiento disponibles en diferentes regiones del país $(7,8)$.

Existe consenso en la literatura que una paciente con cáncer de ovario avanzado debe idealmente ser sometida a cirugía primaria y luego recibir quimioterapia (3). En dicha cirugía el objetivo es alcanzar el menor residuo tumoral posible, es decir la citoreducción óptima, a fin de reducir la carga tumoral con la cual posteriormente deba lidiar la quimioterapia. La definición de citoreducción ha evolucionado en el tiempo hasta alcanzar niveles de residuo menor de $1 \mathrm{~cm}$ o residuo microscópico $(4,5)$. Tal límite de lo óptimo, ha demandado por parte de los ginecólogos oncólogos un incremento de sus destrezas quirúrgicas a fin de poder realizar cirugía ultraradical (9). En centros donde se ha logrado superar la barrera del 60 a $70 \%$ de casos óptimamente citoreducidos, la cirugía no se limita a la histerectomía total, anexectomía bilateral, omentectomía infracólica y remoción de implantes, situación que ocurre actualmente en muchos lugares de nuestro país. Tal magnitud de cirugía solo logra citoreducir adecuadamente no más de un 20 a 30\% de los casos. Los estándares actuales demandan que el cirujano remueva tumor más allá de la pelvis, en particular en el abdomen superior. Es por 


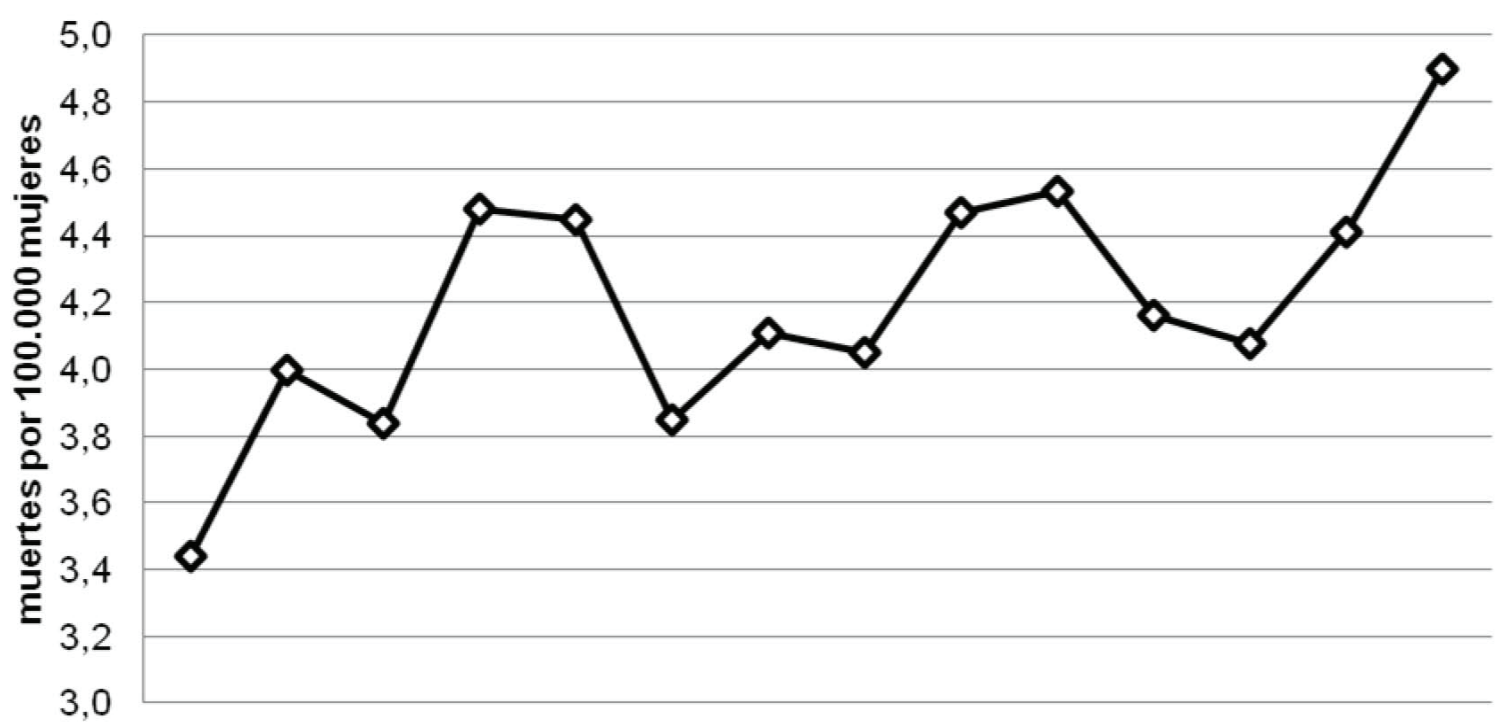

19971998199920002001200220032004200520062007200820092010

Figura 1. Evolución de la tasa de mortalidad por cáncer de ovario en la mujer chilena entre los años 1997 y 2010. (Fuente: Anuarios de Estadísticas Vitales, INE).

ello que la cirugía ultraradical implica efectuar resecciones intestinales únicas o múltiples, remover el diafragma, realizar esplenectomía, pancreatectomía distal, hepatectomía parcial, por nombrar las más frecuentes $(10,11)$. Tal complejidad quirúrgica demanda entrenamiento adicional del ginecólogo oncólogo y exposición a un número mínimo de casos al año (a fin de minimizar las complicaciones), mayor tiempo en pabellón (alrededor de 7 a 9 horas por cirugía en equipos expertos), necesidad de uso de insumos quirúrgicos de mayor costo (ej. suturas mecánicas intestinales), de contar con el apoyo multidisciplinario de otros especialistas para el manejo perioperatorio de complicaciones inherentes a la complejidad de estos casos y donde el cuidado intensivo y el apoyo nutricional juegan un papel central (11).

Respecto de la quimioterapia, los estándares actuales demandan una complejidad también mayor. Además de usar el esquema basado en carboplatino más paclitaxel, existen variaciones en su forma y vía de administración dependiendo de la condición que la enfermedad y la paciente tienen al momento de decidir su uso (3-6). En aquellas pacientes que se logra la citoreducción óptima, idealmente a nivel microscópico, la mejor opción es el uso de quimioterapia endovenosa e intraperitoneal (4-6). Alternativamente es posible administrar quimioterapia endovenosa exclusiva en dosis densa (12). Para pacientes con enfermedad no citoreducible y de mal pronóstico es posible considerar la adición de agentes biológicos como el antiangiogénico bevacizumab (4). Al momento de la recurrencia, dependiendo del intervalo libre de enfermedad es posible utilizar nuevamente esquemas basados en platino (si el intervalo es mayor a 6 meses) o terapias con monodroga o asociada a agentes biológicos (3-6). La diversidad de esquema hace necesario que el oncólogo médico disponga de todo el arsenal terapéutico, de las distintas opciones de administración, tanto en régimen ambulatorio u hospitalizado. Todo ello genera costos elevados que la institucionalidad o el paciente deben asumir.

No cabe duda que el cáncer de ovario epitelial es una enfermedad catastrófica, tanto en lo humano como en lo económico, y por ende resulta indispensable a fin de lograr mejorar la calidad de vida y las expectativas de sobrevida de la mujer afectada, que se establezcan medidas estatales que garanticen tanto el tratamiento adecuado como la protección financiera del individuo.

Recientemente, a comienzos de febrero de 2013, el Ministro de Salud en ejercicio, Dr. Jaime Mañalich, anunció, a través de los medios de comunicación, la incorporación de 11 nuevas patologías al plan AUGE (Acceso Universal Garantías Explícitas), elevando a 80 el número de patologías con cobertura. Dentro de las nuevas patologías incorporadas, afortunadamente se incluyó el enfrentamiento diagnóstico y terapéutico del cáncer de ovario epitelial en sus distintas etapas (13). Sin lugar a dudas, tal incorporación constituye un logro 
y avance sanitario, que permitirá mejorar las opciones de diagnóstico y tratamiento que recibe actualmente nuestra población afectada.

Tal como establece en sus artículos № 2 y 4 la ley AUGE (№19.966) de la República de Chile sobre el régimen general de garantías, instrumento de regulación sanitaria, todo beneficiario de las leyes № 18.469 (FONASA) y № 18.933 (Isapres), las mujeres tendrán garantías explícitas de acceso, de oportunidad, de protección financiera y de calidad, para el tratamiento del cáncer de ovario epitelial. En términos generales establece que frente a la sospecha de un cáncer de ovario, su diagnóstico mediante biopsia deberá ocurrir antes de 30 días, que frente a la necesidad de quimioterapia esta se deberá iniciar antes de 30 días de confirmado el diagnóstico o de realizada la cirugía, y que el seguimiento debe iniciarse antes de 60 días de finalizado el tratamiento. Será apartir del $1^{\circ}$ de julio de 2013 cuando entrará en vigencia el así llamado "plan AUGE 80", que incorpora al cáncer de ovario dentro de las patologías con garantías explícitas.

Para cumplir lo establecido por ley, las autoridades del Ministerio de Salud convocaron durante los últimos años a especialistas de las diferentes áreas a fin de desarrollar una guía clínica que permitiera protocolizar el manejo de esta patología poniendo énfasis en la calidad de las prestaciones (14). Dicha guía evolucionó en el tiempo y a través de 5 años de desarrollo ha ido paulatinamente incorporando los avances ocurridos, permitiendo garantizar estándares de manejo similares a los aplicados en EEUU y Europa (ver Figura 2 con flujograma del proceso clínico de la paciente con cáncer de ovario epitelial).

La ejecución de dicha guía pretende contribuir a cuatro objetivos centrales:

1. Estandarizar el proceso de atención de las mujeres afectadas con cáncer de ovario epitelial.

2. Aumentar la sobrevida global actual, considerando como línea de base conocida el año 2008.

3. Mejorar la calidad de vida de las personas tratadas.

4. Reducir la mortalidad por causa país, ajustada por sexo, considerando como línea de base el año 2008.

Para evaluar el cumplimiento de la guía se han propuesto como indicadores la tasa de infección postoperatoria, la letalidad operatoria y postoperatoria, la latencia para inicio de quimioterapia y la sobrevida global y por etapas a 2 y 5 años.

La implementación de la guía requiere de una canasta AUGE acorde al nivel de prestaciones propuestas. Probablemente ahí resida uno de los primeros problemas al momento de garantizar la calidad de atención para esta patología. Al analizar la canasta se observan deficiencias en el ámbito del diagnóstico y del tratamiento que pueden dificultar el alcance de las metas propuestas. A modo de ejemplo, no se incluyen prestaciones como: estudio endoscópico digestivo alto y bajo para condiciones que forman parte del diagnóstico diferencial, proceso diagnóstico que la guía clínica propone llevar a cabo cada vez que sea necesario. Tampoco se contempla la cobertura de procedimientos quirúrgicos que permitan garantizar la citoreducción óptima (ej. apendicetomía) y la instalación de catéter de quimioterapia intraperitoneal o el uso de quimioterapia asociada a agentes biológicos en casos de cáncer de ovario no citoreducible o recurrente y resistente a platino. Sin lugar a dudas, los avances en el área y las necesidades surgidas de la puesta en práctica de la guía, demandaran la necesidad de modificar y ampliar la cobertura de prestaciones de la canasta original, tal como ya ha ocurrido en una serie de patologías ya incluidas en el AUGE, tal es el caso de la canasta de prestaciones para cáncer de cuello uterino.

Preocupa la referencia oportuna de la paciente correcta. Para ello es necesario que a nivel primario, tanto matrona como ginecólogo general, estén debidamente capacitados de tal forma que conozcan con claridad los criterios de sospecha que ameritan la referencia expedita de una paciente sugerente de tener un cáncer de ovario. El mayor problema puede presentarse con la referencia injustificada de pacientes con tumor anexial complejo que no reúnen todos los criterios y que pudiesen congestionar las vías de derivación. Aquí es trascendental contar con exámenes de diagnóstico confiables que apoyen al clínico en su toma de decisión (ej. informe ecográfico que establezca con claridad criterios de sospecha e incorpore score de riesgo como GIRADS o las reglas propuestas por la IOTA) $(15,16)$. Aquí juegan un rol relevante las sociedades científicas, las universidades y otras instituciones encargadas de la educación de profesionales de la salud. Es su deber mantenerlos actualizados a través de cursos de educación continua donde el énfasis esté en el uso correcto de las herramientas de diagnóstico y el conocimiento de criterios de referencia. No saturar el sistema contribuirá a cumplir con las garantías de acceso y de oportunidad.

Un problema que se avizora ocurrirá en el ejercicio práctico, es el que resulta de la no inclusión de otros tipos histológicos de cáncer de ovario (Señora: usted tiene cáncer de ovario pero no es el del AUGE). En Chile el 70 a $80 \%$ de los casos de cáncer de ovario corresponden a cáncer de ovario epitelial. Sin embargo, el $20 \%$ restante incluye otras variedades como el cáncer de ovario germinal (ej. teratoma inmaduro), del estroma gonadal específico (ej. tumor de la granulosa), del estroma gonadal no específico (ej. sarcoma) y los tumores de origen metastásico (17). Para algunos de ellos, su cobertura podrá estar garantizada por el origen del tumor primario (ej. mama, colon) o por el grupo etario en que se presenta (ej. tumores germinales en mujeres menores de 


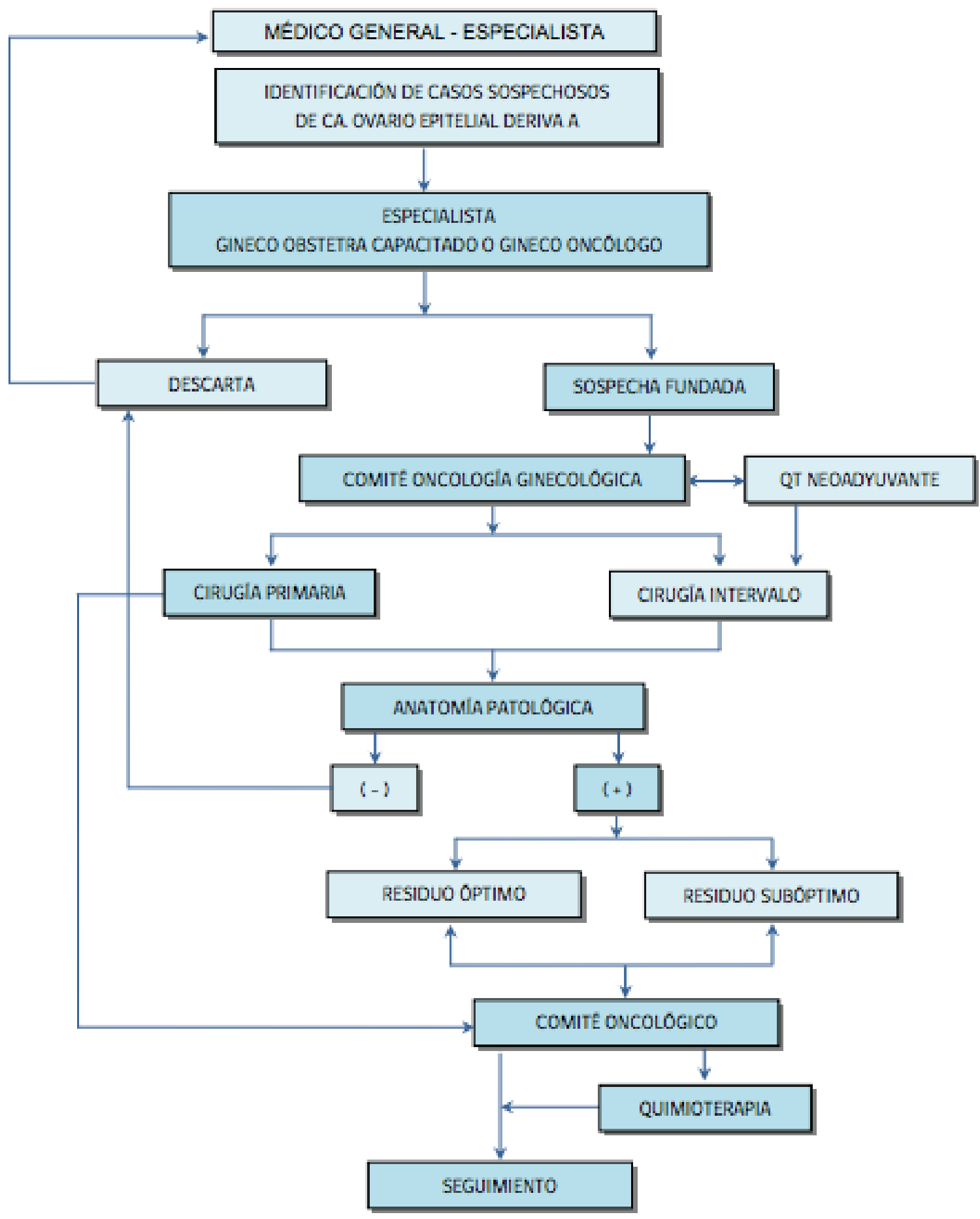

Figura 2. Flujograma del proceso clínico de la guía clínica AUGE en cáncer de ovario epitelial (Fuente: Guía Minsal Cáncer de Ovario Epitelial, 2013, pág. 5). http://www.minsal.cl/portal/url/item/db835d0231a0115fe0400101640126b7.pdf 
15 años). Sin embargo, existe un grupo de pacientes que quedarán potencialmente excluidas. Aunque se trata de un número reducido, habrá algunos casos en los cuales el médico tratante deberá explicar a la paciente que tiene un cáncer de ovario pero que este no está sujeto a las garantías explícitas. Por ello tanto su tratamiento quirúrgico como farmacológico deberá ser cubierto de manera tradicional por el sistema previsional que ella posea. Ojalá en el futuro, estos casos también sean incorporados y la cobertura se haga extensiva a todo cáncer de ovario, independiente de su estirpe.

Preocupa también, el contar con la infraestructura que garantice la resolución quirúrgica oportuna y de calidad. Para ello es necesario contar con acceso a laparoscopia en todo centro que participe del proceso diagnóstico, horas de pabellón y anestesia que permitan resolver en el tiempo requerido cada caso que amerite cirugía primaria ultra radical, sin que ello afecte la resolución de otras patologías no AUGE por parte del especialista. Se requiere además de unidades de cuidado de paciente crítico donde los médicos reciban a estas pacientes sin que existan restricciones producto de la limitación de camas disponibles y donde el triage a veces genera conflictos ético-clínicos, donde la expectativa o posibilidades de sobrevida a veces se transforma en un determinante mayor.

Un desafío mayor es lograr establecer una red de derivación que ofrezca estándares de calidad de atención en cualquier parte del país. En la actualidad no todas las regiones cuentan con infraestructura, recursos económicos y humanos que permitan garantizar lo que la ley exige. Es universalmente aceptado que los mejores resultados en el manejo de patologías de alta complejidad, dentro de las cuales califica el cáncer de ovario, se logran cuando el manejo se centraliza en lugares que cuentan con toda la tecnología y la experiencia. Equipos experimentados permiten maximizar el éxito terapéutico y minimizar las complicaciones y costos asociados a la atención (18). Es deber de la autoridad garantizar que cada región cuente con centros de alta complejidad, con el debido equipamiento y con personal entrenado. En este último punto es deber de las instituciones formadoras garantizar que los nuevos ginecólogos oncólogos sean capaces de efectuar los procedimientos quirúrgicos que esta patología exige, en especial cirugía ultra radical. Así también que los oncólogos médicos manejen la diversidad de esquemas de quimioterapia y terapia paliativa que para esta condición han mostrado ventajas en sobrevida y calidad de vida, respectivamente. Esta fuera de todo cuestionamiento que si un centro no cuenta con tales capacidades es preferible que la paciente sea referida a un centro donde se le ofrezca la calidad de tratamiento que el caso amerita o en su defecto, cuando la derivación no es posible, que se privilegie la neoadyuvancia como alternativa de tratamiento más segura.

Por último, es indispensable que FONASA disponga de los recursos económicos para garantizar el acceso a las prestaciones que se requieren, a fin de lograr calidad en el tratamiento con contención de costos para la mujer afectada. Al tratarse de una enfermedad cuya prevalencia aumenta después de la sexta década de la vida y teniendo en cuenta la dinámica migratoria que se observa a esta edad, desde un sistema previsional a otro (desde Isapre a FONASA), es de esperar que la mayoría de las mujeres afectadas por esta condición accedan al AUGE a través de FONASA. El estado debe ser garante de equidad, en términos de calidad de tratamiento, independiente del copago que deba realizar o del sistema previsional del cual sea beneficiaria la mujer afectada.

No cabe duda que la incorporación del cáncer de ovario es un avance sanitario necesario, agradecido particularmente por cada mujer afectada, por su familia y por el equipo médico. Tal medida evita o limita la catástrofe económica y social que este tipo de enfermedades desencadena. Sin embargo, exigir calidad genera desafíos y potenciales dificultades, que tanto la paciente como el equipo médico, y en consecuencia los entes contralores, deberán estar atentos de pesquisar e informar oportunamente a las autoridades gubernamentales. Todo ello con el fin de contribuir a su pronta rectificación, solución y al mejoramiento continuo de las prestaciones incluidas en el plan.

Dr. Mauricio Cuello F. Ginecólogo Oncólogo. División de Obstetricia y Ginecología. Facultad de Medicina. Pontificia Universidad Católica de Chile

\section{REFERENCIAS}

1. Ferlay J, Shin BF, Bray F, Mathers C, Parkin DM. Globocan 2008 v2.0, Cancer Incidence and Mortality Worldwide: IARC Cancer Base №. 10 [internet]. Lyon, France. In: International Agency for Research on Cancer; 2010.

2. Lowe KA, Chia VM, Taylor A, O'Malley C, Kelsh M, Mohamed $\mathrm{M}$, et al. An international assessment of ovarian cancer incidence and mortality. Gynecol Oncol 2013;130:107-14.

3. Berek JS, Crum C, Friedlander M. Cancer of the ovary, fallopian tube, and peritoneum. Int J Gynaecol Obstet 2012;119(Suppl 2): S118-29.

4. Goff BA. Advanced ovarian cancer: what should be the standard of care? J Gynecol Oncol 2013;24:83-91.

5. Coleman RL, Monk BJ, Sood AK, Herzog TJ. Latest research and treatment of advanced-stage epithelial ovarian cancer. Nat Rev Clin Oncol 2013;10:211-24. 
6. Gardner GJ, Jewell EL. Current and future directions of clinical trials for ovarian cancer. Cancer Control 2011;18:44-51.

7. Encuesta Nacional de Salud ENS Chile, 2009-2010. Santiago: MINSAL; 2010.

8. MINSAL. Esperanza de vida al nacer (en años), por período y sexo. Chile, 1950-2025. Disponible en: http://deis.minsal.cl/deis/ev/esperanza_de_vida/index.asp

9. Wakabayashi MT, Lin PS, Hakim AA. The role of cytoreductive/debulking surgery in ovarian cancer. J Natl Compr Canc Netw 2008;6:803-10; quiz 811.

10. Chang SJ, Bristow RE. Evolution of surgical treatment paradigms for advanced-stage ovarian cancer: redefining 'optimal' residual disease. Gynecol Oncol 2012;125:483-92.

11. Chi DS, Franklin CC, Levine DA, Akselrod F, Sabbatini $P$, Jarnagin WR, et al. Improved optimal cytoreduction rates for stages IIIC and IV epithelial ovarian, fallopian tube, and primary peritoneal cancer: a change in surgical approach. Gynecol Oncol 2004;94:650-4.

12. Katsumata N, Yasuda M, Takahashi F, Isonishi S, Jobo T, Aoki D, et al. Dose-dense paclitaxel once a week in combination with carboplatin every 3 weeks for advanced ovarian cancer: a phase 3, open-label, randomised controlled trial. Lancet 2009;374:1331-8.

13. MINSAL. AUGE 80. Santiago, Chile; 2013. Disponible en: http://www.minsal.cl/portal/url/page/minsalcl/g_ gesauge/auge80.html

14. MINSAL. Guía Clínica AUGE 'Cáncer de Ovario Epitelial'. Santiago: MINSAL; 2013. Disponible en: http://www.minsal.cl/portal/url/item/db835d0231a0115fe0400101640126b7.pdf

15. Amor F, Alcazar JL, Vaccaro $\mathrm{H}$, Leon M, Iturra A. GI-RADS reporting system for ultrasound evaluation of adnexal masses in clinical practice: a prospective multicenter study. Ultrasound Obstet Gynecol 2011;38:450-5.

16. Timmerman D, Valentin L, Bourne TH, Collins WP, Verrelst $\mathrm{H}$, Vergote $\mathrm{I}$, et al. Terms, definitions and measurements to describe the sonographic features of adnexal tumors: a consensus opinion from the International Ovarian Tumor Analysis (IOTA) Group. UItrasound Obstet Gynecol 2000;16:500-5.

17. Cuello M, Merino P, Etchegaray A, Ortega JP, Pomés $\mathrm{C}$, Barrena $\mathrm{N}$, et al. Distribución de la patología anexial en la mujer chilena: experiencia de la Universidad Católica de Chile. Rev Chil Obstet Ginecol 2004;69:429-40.

18. Bristow RE, Zahurak ML, Diaz-Montes TP, Giuntoli $R L$, Armstrong DK. Impact of surgeon and hospital ovarian cancer surgical case volume on in-hospital mortality and related short-term outcomes. Gynecol Oncol 2009;115:334-8. 